\title{
8.8A THE DISCRETE PROLATE SPHERIODAL FILTER AS A DIGITAL SIGNAL PROCESSING TOOL
}

\author{
J. D. Mathews*, J, K. Breaka11**, and G. K. Karawas*
}

*Department of Electrical Engineering and Applied Physics, Case Institute of Technology, Case Western Reserve University, Cleveland, OH 44106

**Lawrence Livermore National Laboratories, P.0.B. 808, Livermore, CA 94550 ABSTRACT

The discrete prolate spheriodal (DPS) filter is one of the class of nonrecursive finite impulse response (FIR) filters. The DPS filter, first introduced by TUFTS and FRANCIS $(1970)$, is superior to other filters in this class in that it has maximum energy concentration in the frequency passband and minimum ringing in the time domain. We give a mathematical development of the DPS filter properties, provide information required to construct the filter, and compare the properties of this filter with those of the more commonly used filters of the same class. We note that use of the DPS filter allows for particularly useful statements of data time/frequency resolution "cell" values and that overall it forms an especially useful tool for digital signal processing.

\section{INTRODUCTION}

The reduction of digitized data to final form usually involves what is broadly described as digital filtering. This process may be applied in either the domain in which sampling occurs (e.g., time or space) or in the corresponding transform domain (e.g., frequency or spatial frequency). The net result of digital filtering is always to "smooth" or restrict the frequency content of the data. However, it is often very important that this smoothing or filtering process be done with minimum and well-understood distortion to the results in both domains. An example of an often misused filter is the equal weight ("square") running average which "smooths" in the domain of application but results in a $(\sin x / x)^{2}$ like passband shape in the transform domain. The $(\sin x / x)^{2}$ behavior may have unacceptable sidelobe levels and/or spacing.

The difficulty of minimizing distortion due to filtering starts with a statement of the criterion for judging when a filter is most satisfactory. We will follow the approach of TUFTS and FRANCIS (1970), PAPOULIS and BERTRAN (1972), and SLEPIAN (1978) in which an optimum finite (length) impulse response (FIR) non-recursive filter is derived on the basis of maximum energy concentration in the passband of the filter relative to energy in the total bandpass up to the Nyquist frequency $\omega_{N}(=2 \pi / 2 \tau$ with $\tau$ the sampling interval). Choice of this energy concentration criterion leads to filters described in terms of discrete prolate spheriodal sequences and wave functions. These sequences and wave functions have many desirable properties which were first investigated in the continuous case by SLEPIAN and POLLAK (1961), LANDAU and POLLAK (1961, 1962), and SLEPIAN (1964).

This paper is basically tutorial in that our major goals are to demonstrate the usefulness of FIR filters based on maximum passband energy concentration and to supply the information necessary for the "construction" of these filters. We do however introduce several apparently new results which prove useful in numerically solving the matrix equations which describe the filter. In the following section we develop the general equations for symetric FIR digital filters and then obtain solutions based on the energy concentration criterion. Next we demonstrate that these filters have features which are desirable 
especially when compared with more traditional filters, and finally, we present a summary and conclusions,

\section{MATHEMATICAL DERIVATIONS}

\section{(a) A General Non-Recursive FIR Filter}

Using the time and frequency domains, a tapped delay line implementation of a non-recursive FIR filter acting on analog signal $x(t)$ is shown schematically in Figure 1. The resultant output signal has the form

$$
y(t)=\sum_{n=-K}^{K} a_{n} x(t-n \tau)
$$

where the coefficients $a_{n}$ are real and where the time reference is, for later convenience, at the center of the delay line. Assuming that the Fourier transform of $x(t) \leftrightarrow X(\omega)$ exists we Fourier transform (1) and find

where

$$
Y(\omega)=H(\omega) \cdot X(\omega)
$$

$$
H(\omega)=\sum_{n=-K}^{K} a_{n} e^{-j n \omega \tau}
$$

is the "voltage" transfer function and $j=\sqrt{-1}$. The corresponding "power" transfer function is

$$
\begin{aligned}
S(\omega) & =H(\omega) \cdot H^{*}(\omega)=\sum_{n, m=-K}^{K} a_{n} a_{m} e^{-j(n-m) \omega \tau} \\
& =\sum_{n=-K}^{K}\left[a_{n}^{2}+2 \sum_{m=-K}^{n-1} a_{n} a_{m} \cos (n-m) \omega \tau\right]
\end{aligned}
$$

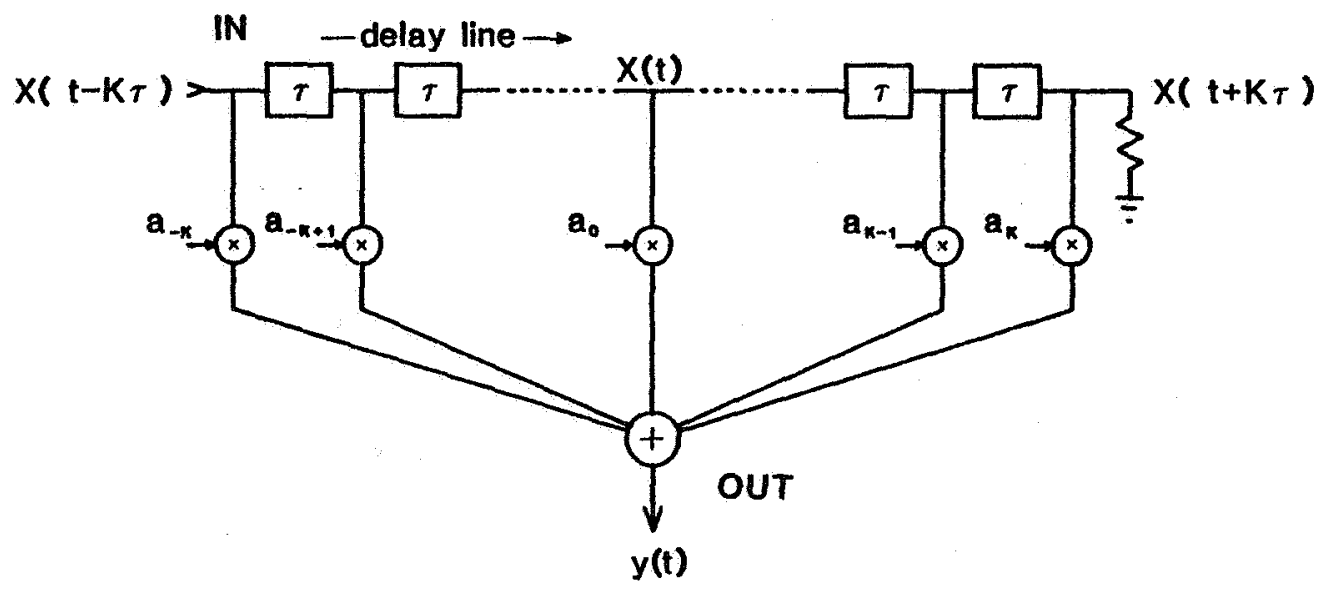

Figure 1. Block diagram of the non-recursive FIR filter described by Equation 1. 
while the inverse Fourier transform of (3) is

$$
h(t)=\sum_{n=-K}^{K} a_{n} \delta(t-n \tau)
$$

the filter impulse response with $\delta\left(t^{\prime}\right)$ the unit impulse. Note that $h(t)$ convolved with $x(t)$ yields equation ( 1$)$.

The filter described by (3) or (5) becomes a digital filter if we uniformly and instantaneously sample $y(t)$ at intervals of $\tau$ time. Then (1) becomes

$$
y_{j}=\sum_{n=-k}^{K} a_{n} x_{j-n}
$$

where the index $j$ refers to consecutive members of the set of sampled signals. If we restrict $x(t)$ to be bandlimited to the Nyquist frequency $(2 \pi / 2 \tau)$ or less, then the sampling/filtering process occurs without aliasing.

(b) Choice of Coefficients

Equations (3) or (5) dtocribe the effects of the filter on an input signal given a particular set of coefficients $\{a\}$. These coefficients are of ten chosen such that the digital filter characteristics are similar to one of various common analog filters (e.g., the "ideal" filter). The process of synthesizing these filter characteristics often involves smoothing (windowing) of the resultant coefficient sequence to suppress ringing (BLACKMAN and TUKEY, 1958; HAMMING, 1977).

As mentioned in the introduction, we propose to choose the sequence $\left\{a_{n}\right\}$ by maximizing the energy concentration of $s(\omega)$ (equation (4)) in some interval $\left[-\omega_{c}, \omega_{c}\right]$ on $\left[-\omega_{n}, \omega_{n}\right]$ where $\omega_{c}$ is the filter "cutoff" frequency. Thus let

$$
\alpha=\int_{-\omega_{c}}^{\omega} c(\omega) d \omega / \int_{-\omega_{n}}^{\omega} n s(\omega) d \omega
$$

and $f$ ind $\left\{a_{n}\right\}$ such that $\alpha$ is maximized. Substituting equation (4) for $s(w)$ in (7) and performing the integrations yield

$$
\alpha=\frac{\sum_{n, m=-K}^{k} a_{n} a_{m} \frac{\sin (n-m) \pi \varepsilon}{(n-m) \pi}}{\sum_{n=-K}^{K} a_{n}^{2}}
$$

where $\varepsilon=\omega_{c} / \omega_{n}$. If we define $\left\{a_{n}\right\}$ as elements of vector a then (8) becomes

$$
\underline{a}^{T} \cdot \stackrel{E}{=K}_{K} \cdot \underline{a}-\alpha \underline{a}^{T} \cdot \underline{a}=0
$$

where each $\underline{\text { a has }}(2 K+1)$ elements and the elements of matrix $\underline{\underline{E}}_{K}$ are

$$
\begin{aligned}
& \mathrm{e}_{\mathrm{mn}}=\frac{\sin (\mathrm{n}-\mathrm{m}) \pi \varepsilon}{(\mathrm{n}-\mathrm{m}) \pi} \\
& -\mathrm{K} \leq \mathrm{m}, \mathrm{n} \leq \mathrm{K}
\end{aligned}
$$

We are thus searching for a such that $\alpha$ is maximum. From Lagrange 
multiplier theory, it is clear that (9) is satisfied by the set of $(2 \mathrm{~K}+1)$ orthonormal eigenvectors $\underline{a}^{(i)}$ and the corresponding eigenvalues $\lambda_{i}$ of the system

$$
\left(\underline{\underline{E}}-\lambda_{i \underline{I}}\right) \cdot \underline{a}^{(i)}=0
$$

where $I$ is the identity matrix. We choose the elements of $a^{(0)}$ the eigenvector associated with $\lambda_{0}$, the largest eigenvalue, as coefficients of our "optimum" filter. That is, $\underline{a}(0)$ maximizes equation (8) with $\alpha=\lambda_{0}$ for the $K$ and $\varepsilon$ (i.e., $\omega_{c}$ ) values in question.

SLEPIAN (1978) refers to these eigenvectors and corresponding $H(\omega)$ 's as discrete prolate spheriodal sequences and wave functions, respectively. He demonstrates that if we order the eigenvalues according to magnitude,

$$
1>\lambda_{0}>\lambda_{1}>\ldots>\lambda_{2 K}>0
$$

and if $a_{n}$ (i) is the $n^{\prime}$ th element of the eigenvector corresponding to $\lambda_{i}$ then

$$
\sum_{n=-K}^{k} a_{n}^{(i)} a_{n}(j)=\delta_{i j}= \begin{cases}1 & i=j \\ 0 & i \neq j\end{cases}
$$

Letting $b_{n}(j)$ be the $n^{\prime}$ th element of the eigenvector corresponding to eigenvalue $\lambda_{j}$ calculated for $\omega_{c}^{\prime}=\omega_{N}-\omega_{c}$ then (SLEPIAN, 1978; section 2.2)

$$
\left.\begin{array}{l}
a_{-n}^{(i)}=(-1)^{i} a_{n}^{(i)} \\
a_{n}^{(i)}=(-1)^{|n|} b_{n}(2 K-i) \\
|n| \leq k
\end{array}\right\}
$$

Slepian also notes that the discrete prolate spheroidal wave functions $U(\omega)$ satisfy a Sturm-Liouville equation of the form

$$
\frac{d}{d \omega}[\cos \omega \tau-\cos \pi \varepsilon] \frac{d U}{d \omega}+\tau^{2}[K(K+1) \cos \omega \tau-\theta] U=0
$$

where $\theta$ is the eigenvalue (different from $\lambda$ ) corresponding to a particular wave function and all other parameters are as before. Substituting $H(\omega) \quad(=U)$ from (3) into (12) we find

$$
\left(\underline{E}_{K}^{\prime}-\theta_{i} \underline{\underline{I}}\right) \cdot \underline{a}^{(i)}=0
$$

where for $|n| \leq k$

$$
\mathrm{e}_{\mathrm{mn}}^{\prime}= \begin{cases}\frac{1}{2}(\mathrm{k}+\mathrm{m})(\mathrm{k}-\mathrm{m}+1) & \mathrm{m}=\mathrm{n}-1 \geq 0 \\ \mathrm{~m}^{2} \cos \pi \varepsilon & \mathrm{m}=\mathrm{n} \\ \frac{1}{2}(\mathrm{~K}-\mathrm{m})(\mathrm{k}+\mathrm{m}+1) & \mathrm{m}=\mathrm{n}+1 \leq \mathrm{K} \\ 0 \quad \text { otherwise } & \end{cases}
$$

or equivalently

$$
\frac{1}{2}(k+n)(k-n+1) a_{n-1}+\left(n^{2} \cos \pi \varepsilon-\theta_{i}\right) a_{n}+\frac{1}{2}(K-n)(K+n+1) a_{n+1}=0
$$


The matrix $E^{\prime}$ is tridiagonal and thus equation (14) is much easier to solve numerically than equation (11). Equation (14) yields the same normalized eigenvectors as (11) however the corresponding eigenvalues are different although we still seek the eigenvector corresponding to the largest eigenvalue $\theta_{0}$. Since we are interested only in $\underline{a}(0)$ which is symmetric we can essentially halve the size of $\underline{E}^{\prime}$ by invoking symmetry (see equation (12)) which yields

$$
-\theta_{i} a_{0}+K(K+1) a_{1}=0
$$

plus equation (16) with $1 \leq n \leq K$. The compact form of (14) yields only eigenvalues corresponding to "even" eigenvectors and the resultant $\underline{g}$ 's may be normalized such that $\underline{a}^{\mathrm{T}} \cdot \underline{a}=1$.

From equation (14) we find that for $\omega_{0}=0, \theta_{0}=k(k+1)$, and $a_{n}(|n|<k)=$ 1 while for $\omega_{c}=\omega_{N}(i, e ., \varepsilon=1)$ we find $\varepsilon$ using the $\omega_{c}=0$ case and $\left.(12)\right)$ that $\theta_{0}=k^{2}$ and $a_{n}=a_{n-1} \frac{[k-(n-1)]}{K+n}$

$$
1 \leq n \leq k
$$

We observe experimentally that $k(k+1) \geq{ }_{0} \geq R^{2}$ for all $0 \leq \omega_{c} \leq \omega_{N}$ and that

$$
\begin{aligned}
& a_{0}^{(0)}>a_{1}^{(0)}>a_{2}{ }^{(0)}>\ldots>a_{K}{ }^{(0)} \geq 0 \\
& 1 \geq a_{i}^{(0)}\left(\omega_{c}\right) \geq a_{i}^{(0)}\left(\omega_{c}=\omega_{N}\right)
\end{aligned}
$$

where $a^{(0)}\left(\omega_{c}\right)$ refers to the $i^{\prime}$ th component of the eigenvector corresponding to the fargest eigenvalue for $0 \leq \omega_{c} \leq \omega_{N}$. The range constraints on the value of $\theta_{0}$ may be used in finding $\theta_{0}$ from (14).

COMPARISON OF FILTER PROPERTIES

In the previous section we showed that FIR filters with discrete prolate spheriodal sequences as coefficients (see equation (1)) have maximum energy concentration in the passband. These filters are known as discrete prolate spheriodal (DPS) filters and in this section we compare their properties with those of more traditional FIR filters.

These "traditional" filters are all based on various windowing (weighting) functions applied to the coefficients of the infinite Fourier series representation of the ideal low-pass filter. The coefficients of this series are

$$
\begin{aligned}
& \mathbf{c}_{\mathrm{n}}=\frac{\sin \mathrm{n} \pi \varepsilon}{\mathbf{n} \pi} \\
& \mathbf{n}=0, \pm 1, \pm 2, \ldots \\
& \varepsilon=\omega_{\mathrm{c}} / \omega_{\mathrm{n}}
\end{aligned}
$$

and the windowing function $W(n)$ is such that $W(n)=0$ for $|n| 2 K$ thus truncating the series so that the resultant transfer function becomes

$$
H_{W}(\omega)=\sum_{n=-K}^{K} W(n) c_{n} e^{-j n \omega \tau}
$$


The $W(n)(-K<n<K)$ are chosen to minimize in some sense the ringing (Gibb's phenomena) which results from truncation of the series representation of the ideal lowpass filter (HAMMING, 1977; Chap. 5, or RABINER and GOLD, 1975; Chap. 3). In this discussion, we compare the DPS filter with the $H_{W}(\omega)$ (equation (18)) of same length using rectangular, Kaiser, Von Hann, Dolph-Chebyshev, and Hamming windows. The W(n) corresponding to these windows are given or referenced in Table 1 and are discussed in more detail by HAMMING (1977) and RABINER et a1. (1979).

Figures 2 and 3 compare the frequency response (in dB) plotted versus normalized frequency $f$ (Nyquist frequency $f_{N}=0.5$ ) and impulse response envelope plotted versus time in samples for all six filters. For this comparison we choose $K=15$ (total length 31 ) and adjust filter parameters such that the $-3 \mathrm{~dB}$ level of each filter occurs near $f=0.05$. The filters appear in order of, in our opinion, acceptability with the rectangular windowed filter least acceptable and DPS filter most acceptable.

The frequency domain distortion of a signal spectrum is just the filter frequency response as shown in Figures 2 and 3 . However, the time domain distortions due to the impulse responses are not so obvious. In Figure 4, we show the effects of these filters in the time domain on a square wave plus Gaussian distributed noise. As expected, all filters except the DPS filter "ring". The DPS filter "smooths" the input signal while retaining a "smooth" frequency domain behavior.

To aid in understanding the "evolution" of the DPS filter, we show in Figure 5 the DPS filter frequency response characteristics for normalized cutoff frequency $f_{c}=0.2$ as $K$ is increased from 1 ( 3 coefficients) to 8 (17 coefficients). Recall that in each case energy concentration in the passband (f $\leq 0.2$ ) is the maximum possible for the number of coefficients used.

\section{SUMMARY AND CONCLUSION}

We have investigated the properties of the discrete prolate spheroidal filter relative to other common FIR filters. To accomplish this comparison we first dexive the general properties of FIR filters and then specialize these

Table 1. Weighting coefficients of various windows $(W(|n|>K)=0$ for $a 11$ windows

RECTANGULAR WINDOW: $W(\mathrm{n})=1$

KAISER WINDOW:

\[ W(n)=\frac{I_{0}\left(\alpha \sqrt{1-(n / k)^{2}}\right)}{I_{0}(\alpha)} \]
where $I_{0}(x)=1+\sum_{n=1}^{\infty}\left[\frac{(x / 2)^{n}}{n !}\right]^{2}$

and we use $\alpha=3.395$ (40 $\mathrm{dB}$ minimum stopband attenuation)

VON HANN WINDON: $W(n)=\frac{1+\cos \pi n / K}{2}$

DOLPH-CHEBYSHEV WINDOW: See HELMS (1968) and RABINER et a1. (1979)

HAMMING WINDOW: $W(n)=0.54+0.46 \cos (2 \pi n /(K-1))$ 
properties to the case of maximum energy concentration in the filter passband. The condition of maximum energy concentration gives rise to an eigenvalue problem which has the discrete prolate spheroidal sequences (SLEPIAN, 1978) as eigenvector solutions. The eigenvector corresponding to the largest eigenvalue is used as the coefficient sequence in the DPS filter.

SLEPIAN (1978) also points out that the prolate spheroidal wave functions satisfy a form of the Sturm-Liouville equation. This fact leads to a second eigenvalue/eigenvector problem which is much simpler to solve numerically than the first problem. We note that the elenents of the eigenvector $(a(0))$ corresponding to the largest eigenvalue $\left(\theta_{0}\right.$ or $\left.\lambda_{0}\right)$ are symetric around the center value $\left(a_{0}\right)$ and that

$a_{n-1}$

(0) $\geq a_{n}{ }^{(0)} \geq 0(1 \leq n \leq x)$.
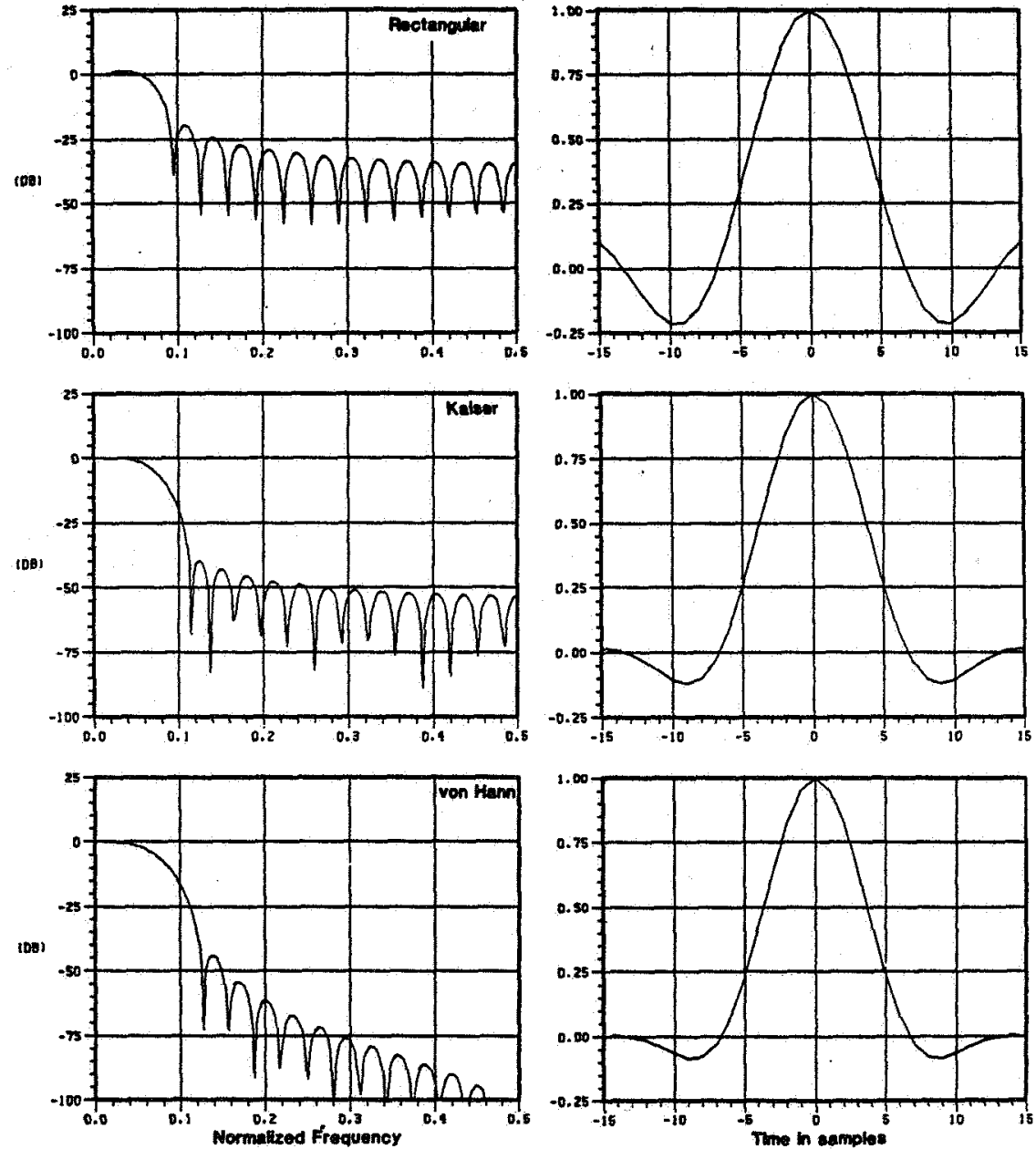

Figure 2. Filter frequency response (dB) plotted versus normalized frequency (Nyquist frequency $\mathrm{f}_{\mathrm{N}^{+}}$).5) and corresponding impulse response envelope plotted versus time in samples for rectangular, Kaiser and von Hann windows of length 31 (see Equation 18 and Table 1). In all cases the normalized $3 \mathrm{~dB}$ frequency is about 0.05 . 
When used, DPS filters have several features which we feel make them superior to more standard FIR filters. Specifically, the DPS filter is "smooth" in both domains and thus distortion of the signal is minimal and easily described in both domains. Thus for example, after removing high frequency noise, a time domain feature may be more easily "restored" by deconvolving the DPS impulse response than by deconvolving the other sin $x / x$ like impulse responses. Another useful feature of DPS filters is that descriptions of time and frequency resolutions of published data can be more readily appreciated and used by the reader. In fact, we suggest that the DPS filter might form a basis for standardization of information concerning the "resolution cells" of data.
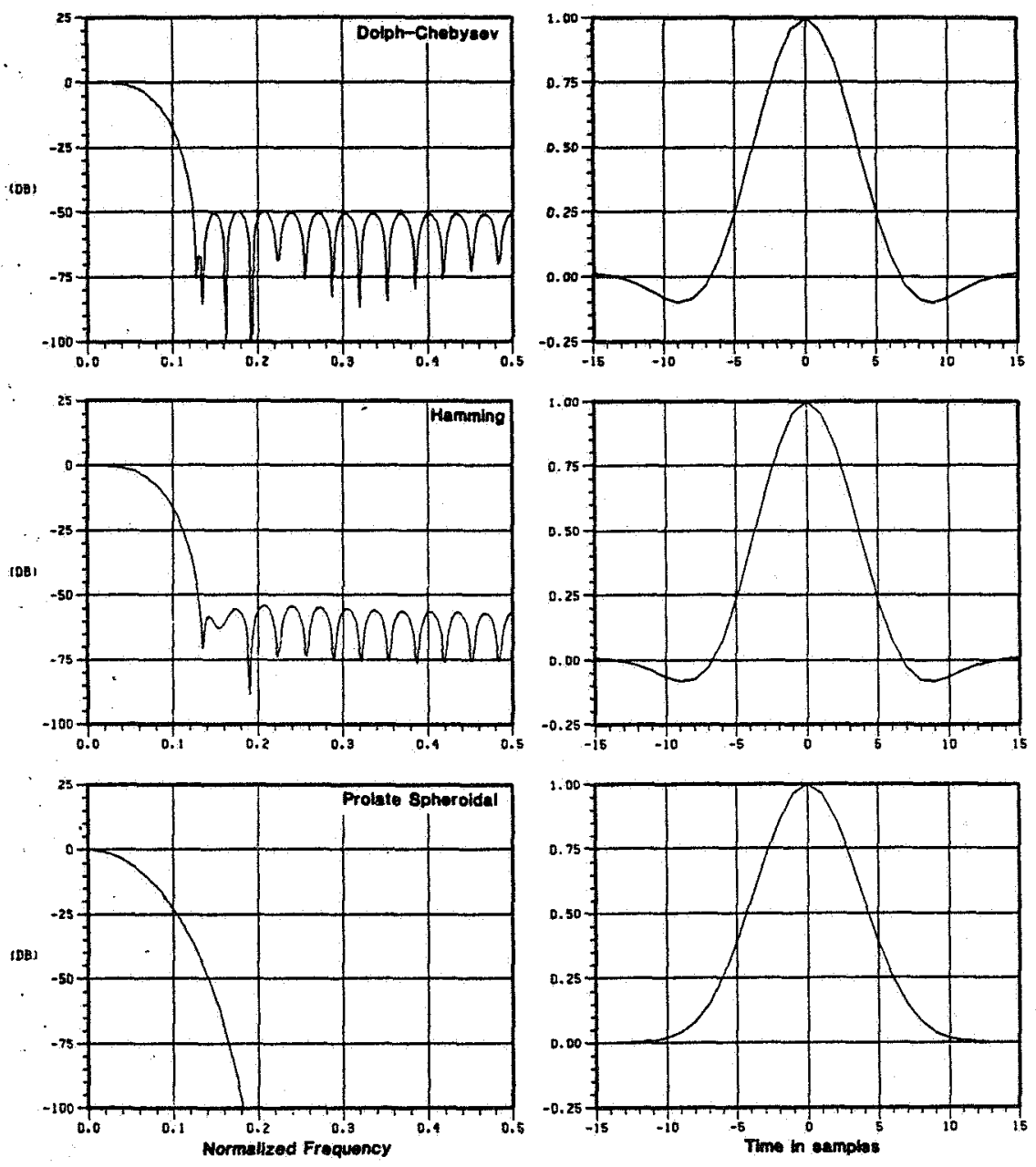

Figure 3. Similar to Figure 2, but for Dolph-Chebysev and Hamming windows and the prolate spheroidal filter. The normalized cutoff frequency of the prolate spheroidal filter is 0.2 . The filters in Figures 2 and 3 are ordered according to overall quality with, in our opinion, the rectangular window filter worst and prolate spheroidal filter best. 

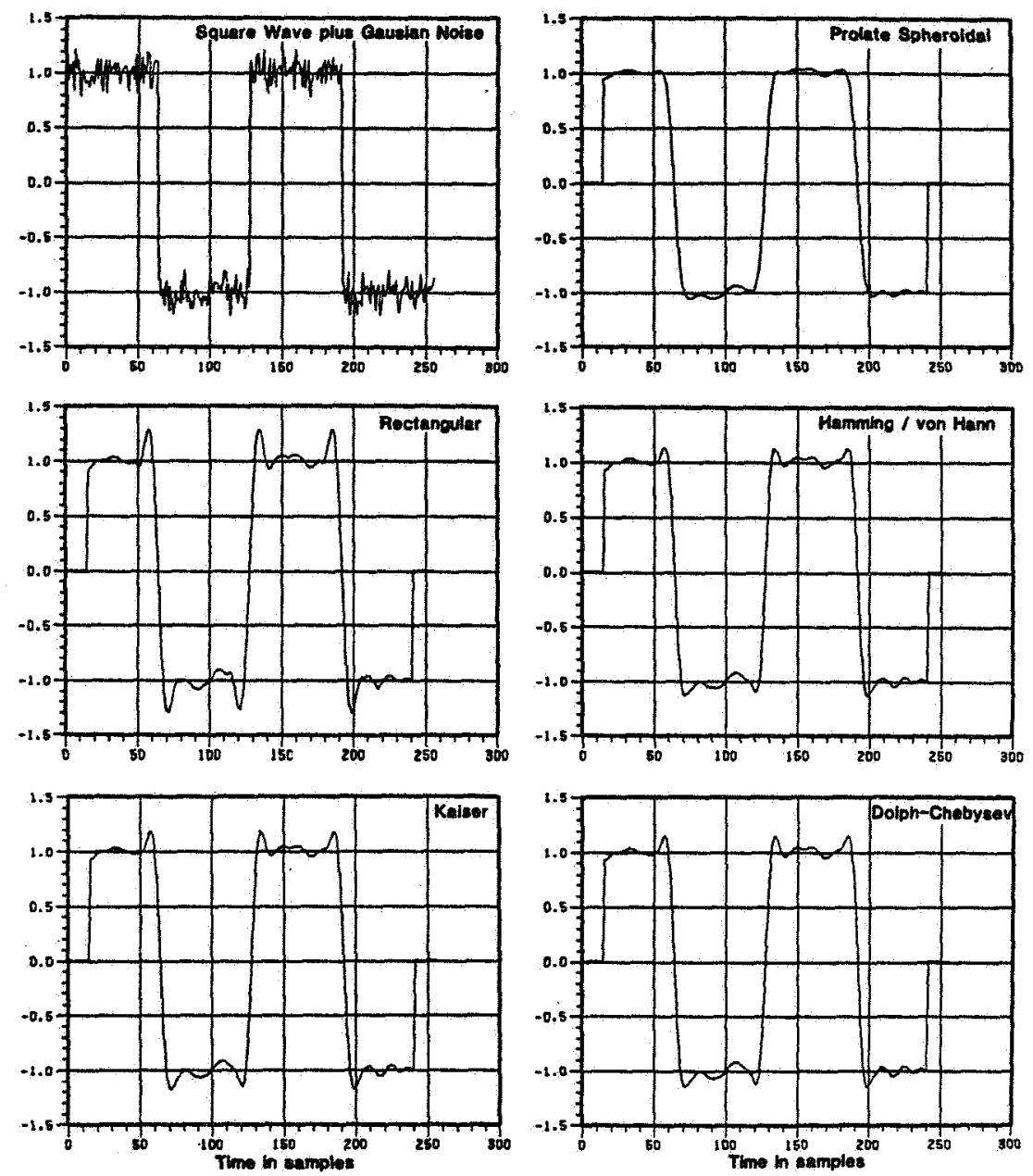

Figure 4. We compare the time domain effects of the various filters on a square wave plus Gaussian distributed noise. Note that all filters except the prolate spheroidal filter "ring" and that the rectangular window filter rings most with the Kaiser window next while the Dolph-Chebysev, Hamming, and von Hann windows are similar in this case.

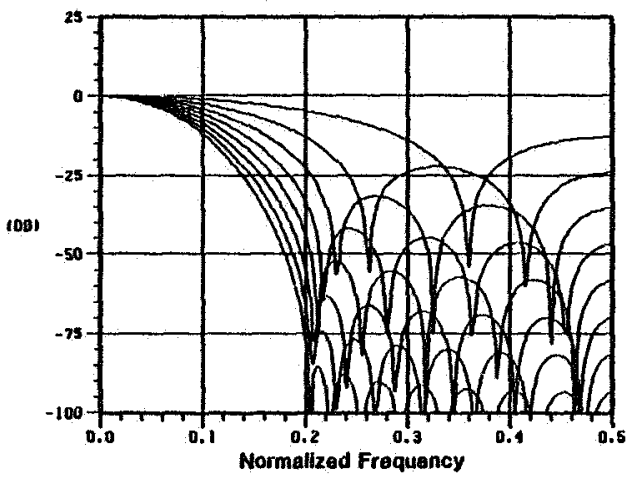

Figure 5. The evolution of the prolate spheroidal filter frequency response as $\mathrm{K}$ increases from 1 through 8 for a normalized cutoff frequency of 0.2 . 


\section{ACKNOWLEDG EMENTS}

This work has been supported by the National science Foundation Atmospheric Sciences Division under grant ATM 79-18379 to the Case Western Reserve University.

\section{REFEREN CES}

Blackman, R. B. and J. W. Tukey (1958), The Measurement of Power Spectra, Dover Publications, New York.

Hamming, R. W. (1977), Digital Filters, Prentice-Hall Signal Processing Series, Prentice-Hall, Englewood Cliffs, New Jersey.

Helms, H. D. (1968), Non-recursive digital filters: Design methods for achieving specifications on frequency response, IEEE Trans. Audio Electroacoust., AU-16, 336-342.

Landau, H. J. and H. O. Pollak (1961), Prolate spheroidal wave functions, Fourier analysis and, uncertainty-II, Bell. Sys. Tech. J., 40, 65-84.

Landau, H. J. and H. O. Pollak (1962), Prolate spheroidal wave functions, Fourier analysis, and uncertainty-III, Bell. Sys. Tech. J., 41, 1295-1336.

Papoulis, A. and M. S. Bertran (1972), Digital filtering and prolate functions, IEEE Trans. Circuit Theory, CT-19, 674-681.

Rabiner, L. R. and B. Gold (1975), Theory and Application of Digital Signal Processing, Prentice-Ha11, Inc., Englewood Cliffs, New Jersey.

Rabiner, L. R., C. A. McGonegal and C. Paul (1979), FIR windowed filter design program - WINDOW, section 5.2, Programs for Digital Signal Processing, IEEE Press, The Institute for Electrical and Electronics Engineers, Inc., New York.

Slepian, D. (1964), Prolate spheroidal wave functions, Fourier analysis, and uncertainty-IV, Bell Sys. Tech. J., 43, 3009-3058.

Slepian, D. (1978), Prolate spheroidal wave functions, Fourier analysis, and uncertainty-V: Discrete case, Bell Sys. Tech. J., 57, 1371-1430.

Slepian, D. and H. O. Pollak (1961), Prolate spheroidal wave functions, Fourier analysis, and uncertainty-I, Bel1 Sys. Tech. J., 40, 43-64.

Tufes, D, W. and J. T. Francis (1970), Designing digital low-pass filters comparison of some methods and criteria, IEEE Trans. Audio and Electroacoustics, AU-18, 487-494. 